\title{
Dosimetric study of the protection level of the bone marrow in patients with cervical or endometrial cancer for three radiotherapy techniques - 3D CRT, IMRT and VMAT. Study protocol.
}

\author{
Agata Jodda ${ }^{1, a}$, Bartosz Urbański ${ }^{2}$, Tomasz Piotrowski ${ }^{1,3}$, Julian Malicki ${ }^{1,3}$ \\ ${ }^{l}$ Department of Medical Physics, Greater Poland Cancer Centre, Poznan, Poland \\ ${ }^{2}$ Department of Radiotherapy and Gynecological Oncology, Greater Poland Cancer Centre, Poznan, Poland \\ ${ }^{3}$ Department of Electroradiology, University of Medical Sciences, Poznan, Poland \\ ${ }^{a}$ E-mail address: agata.jodda@gmail.com
}

\begin{abstract}
Background: The paper shows the methodology of an in-phantom study of the protection level of the bone marrow in patients with cervical or endometrial cancer for three radiotherapy techniques: three-dimensional conformal radiotherapy, intensity modulated radiotherapy, and volumetric modulated arc therapy, preceded by the procedures of image guidance.

Methods/Design: The dosimetric evaluation of the doses will be performed in an in-house multi-element anthropomorphic phantom of the female pelvic area created by three-dimensional printing technology. The volume and position of the structures will be regulated according to the guidelines from the Bayesian network. The input data for the learning procedure of the model will be obtained from the retrospective analysis of imaging data obtained for 96 patients with endometrial cancer or cervical cancer treated with radiotherapy in our centre in 2008-2013. Three anatomical representations of the phantom simulating three independent clinical cases will be chosen. Five alternative treatment plans $(1 \mathrm{x}$ three-dimensional conformal radiotherapy, $2 \mathrm{x}$ intensity modulated radiotherapy and $2 \mathrm{x}$ volumetric modulated arc therapy) will be created for each representation. To simulate image-guided radiotherapy, ten specific recombinations will be designated, for each anatomical representation separately, reflecting possible changes in the volume and position of the phantom components.

Discussion: The comparative analysis of planned measurements will identify discrepancies between calculated doses and doses that were measured in the phantom. Finally, differences between the doses cumulated in the hip plates performed by different techniques simulating the gynaecological patients' irradiation of dose delivery will be established. The results of this study will form the basis of the prospective clinical trial that will be designed for the assessment of hematologic toxicity and its correlation with the doses cumulated in the hip plates, for gynaecologic patients undergoing radiation therapy.
\end{abstract}

Key words: gynaecological cancer; hematologic toxicity; radiotherapy; 3D printing; Bayesian networks; deformable image registration.

\section{Introduction}

Radiation therapy (RT) has been used for treatment of gynaecologic malignancies for over a century. At present, RT can be used as an independent therapy, together with chemotherapy or as complementary treatment following surgery. The main gynaecologic malignancies subjected to RT are: cervical carcinoma, endometrial carcinoma, and vulvar carcinoma.

Conventional radiotherapy (3DCRT) is understood as a method of external beam irradiation during the application of which the dose is delivered using either an arrangement of the AP and PA opposed beams or an arrangement of two pairs of opposed beams, the so called box technique (machine gantry at $0^{\circ}, 90^{\circ}, 180^{\circ}$, and $270^{\circ}$ ). The above-mentioned geometric schemes provide high and homogeneous doses to the tumour. Nevertheless, they also lead to large volumes of healthy tissues receiving high dose of radiation, including organs at risk (OARs), which surround the irradiated area. The attempts to conform dose distribution for such sets of therapeutic beams by adjusting the therapeutic field shape to the target volume using individual shields or multi leaf collimator reduce only slightly doses received by healthy tissues. Thus, the reduction of high doses to organs at risk is unsatisfactory. This limits significantly the total dose that may be administered during 3DCRT. However, the recent advances in radiotherapy techniques have removed some of the limitations of gynaecologic radiotherapy.

One of the more spectacular technological achievements is the introduction of intensity modulated radiation therapy 
(IMRT), which enables a significant reduction of high dose to the area of healthy tissue and organs at risk while retaining homogeneous distribution in the tumour area. In recent years, volumetric modulated arc therapy (VMAT) techniques have gained popularity as, for a number of clinical cases, they ensure an increase in dose distribution conformity when compared to IMRT [1,2].

In the pilot study supported by literature review [3], a retrospective analysis of the IMRT and VMAT plans was performed for patients with endometrial or cervical cancer treated previously by the conventional method (3DCRT). The uniformity of dose distribution in the tumor for IMRT and VMAT techniques has not deteriorated relative to 3DCRT. Nevertheless, the new techniques, in relation to 3DCRT, allow to achieve a significant reduction of high doses in healthy tissues and organs at risk [4-7]. Especially, it ought to be emphasized that IMRT and VMAT decrease the doses to bone marrow located in the hip plates, as evidenced clinically by the reduction of acute hematologic toxicity [8,9].

The clinical benefit from VMAT, in comparison to IMRT, is not yet confirmed. Moreover, modern RT should be preceded by image guidance strategies. The irradiated area consists of several anatomical components, i.e. the tumour, OARs (bladder, rectum, bowel, femoral heads, bone marrow) and other healthy tissues that may move relative to one another during radiotherapy. Modern image guided radiotherapy (IGRT) allows to determine the current position of the tumour and, consequently, provide precise dose delivery in the vicinity of the tumour. The mobility of the tumour in gynaecologic radiotherapy did not correlate with the mobility of the skeletal system. Therefore, the doses absorbed by the bone marrow during the radiotherapy may differ from those calculated during the treatment plan preparation. Therefore, a clinical trial study preceded by basic research should be performed to confirm the potential benefits of the usage of the VMAT techniques in gynaecological radiotherapy.

This paper presents the methodology of the study implemented in our hospital as a dosimetric evaluation of the protection level of the bone marrow in patients with cervical or endometrial cancer for three radiotherapy techniques 3DCRT, IMRT and VMAT.

\section{Methods/Design}

All study tasks will be performed in an in-house multi-element anthropomorphic phantom of the female pelvic area. To create a phantom reflecting the patient's anatomy, input data of the anatomical conditions regarding gynaecological patients were obtained from the retrospective analysis of imaging data gathered from 96 patients treated in our hospital in 2008-2013. The selection criteria for the retrospective data included: (i) endometrial or cervical cancer; (ii) body mass index (BMI) higher that 18.5 (ii) radiation therapy delivered on conventional accelerators (C-arm design) equipped with cone beam CT (CBCT) technology; (iii) CBCT performed not less than in
$25 \%$ of delivered fractions per patient. Patients for whom the break during the RT or re-planning procedure was longer than one week were excluded from the study. The patients were split into three groups according to their BMIs [10]. The groups contained respectively: (A) -43 patients (normal group, $18.5 \leq \mathrm{BMI}<25$ ), (B) -34 patients (overweight group, $25 \leq$ BMI < 30) and (C) -19 patients (obese group, BMI $\geq 30$ ). Detailed analysis of the geometrical conditions of the hip plates, bladder, rectum and tumour will be done for each group. As a result, three representative patients (one for each group) will be chosen as an anatomical pattern during the creation of the phantom.

The main structural component of the anthropomorphic phantom will be form (A) corresponding to the reference volume of the patient's body selected for the first group. It will be a form with a perspex wall filled by water. Outside form (A), two removable overlays (B) and (C) will be placed formed with a solid water. Connection of form (A) and overlay (B) will correspond to the reference volume of the patient's body for the second group and the connection of form (A) and two overlays (B) and (C) will correspond to the reference volume of the patient's body for the third group. Inside the water environment of form (A) a quasi-anatomical elements, such as hip plates, rectum, bladder and tumour, will be placed. The size, shape and volume of the hip plates will depend of the combination of forms (A), (B) and (C). Respectively, we are planning to use three removable hip plate inserts that will be placed rigidly in the water environment of form (A), the replacement of which will depend on the shape of the phantom: $(A)$ or $(A)+(B)$ or $(\mathrm{A})+(\mathrm{B})+(\mathrm{C})$. The rectum and the bladder will be represented by the elastic elements (balloons) that will be filled, respectively, with air and liquid (with a higher electron density than water). The balloons will be rigidly fixed in a few representative anatomical points. The fixation of these elements will enable limited mobility and will not affect potential changes of their volumes, which can be regulated between fractions of irradiation. The rigid element representing the tumour will be placed into the water environment of form (A) to enable all possible movements of the tumour during the treatment.

All solid elements of the phantom will be created using 3D printing technology [11,12] based on anatomical data recovered from the retrospective group of patients. To control the dose delivery, the solid structures (e.g. hip plates, tumour) will have customized holes for the ionization chambers and places for gafchromic inserts. Figure 1 shows the schematic diagram of the phantom project.

Potential recombinations of the shape and positions of the anatomical elements included in the phantom will be designed according to the results obtained from the Bayesian network. The Bayesian network is a graphical class of probabilistic models that raise increasing interest among researchers and clinicians during the simulations of the patient-specific or technology-specific tasks during radiotherapy that are difficult to resolve by conventional methods. 


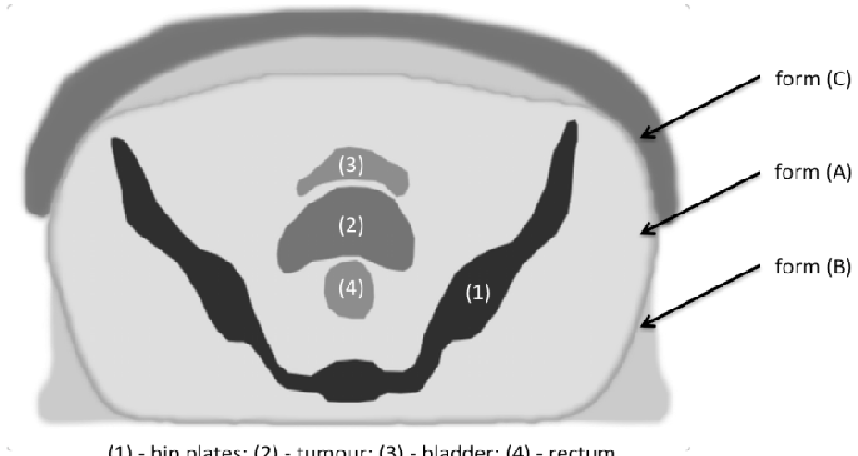

(1) - hip plates; (2) - tumour; (3) - bladder; (4) - recturn

Figure 1. Schematic diagram of the phantom project.

Bayes' theorem proposes the calculation to be performed in order to revise earlier beliefs in the light of new evidence: $\mathrm{P}(\mathrm{X} \mid \mathrm{Y})=\mathrm{P}(\mathrm{X}, \mathrm{Y}) / \mathrm{P}(\mathrm{Y})=\mathrm{P}(\mathrm{Y} \mid \mathrm{X}) / \mathrm{P}(\mathrm{Y}) * \mathrm{P}(\mathrm{X})$. The expression $\mathrm{P}(\mathrm{X} \mid \mathrm{Y})$ in the above formula is the probability of event $\mathrm{X}$ subsidiary of event $\mathrm{Y}$ - and therefore $\mathrm{X}$ is true if $\mathrm{Y}$ has occurred. Based on the causal nature of phenomena, Bayesian network modelling results in a wide range of relationships $[13,14]$. In our case, the network will be used for prediction the anatomical changes occurring during radiation therapy for gynaecologic patients. The learning data for the network will be gathered from CBCT images collected for the same retrospective group of patients that will be used for phantom design and creation. The network will be designed in Matlab environment (MathWorks Inc., Natick, MA, USA) according to the code presented by Murphy [15]. The accuracy of the network results will be tested on currently treated patients for whom CBCT imaging is performed.

A retrospective analysis of imaging data will also be used to determine three anatomical representations of the phantom simulating three independent clinical cases (A, B and C). The selected representations differ from one another in the relative position of the area and volume of the tumour and organs at risk. For each representation, CT scans with a $2 \mathrm{~mm}$ inter-slice distance will be prepared on the Somatom Definition AS scanner (Siemens AG, Erlangen, Germany) and treatment plans will be created in three alternative techniques of radiotherapy (3DCRT, IMRT, VMAT). The delineation will include: tumour, hip plates, femoral heads, bladder, rectum and measurement areas (holes for ionization chambers) located in the tumour and hip plates. All plans will be prepared in the Eclipse treatment planning system for TrueBeam machine equipped with IGRT couch (Varian Medical Systems, Palo Alto, CA, USA). The Anisotropic Analytical Algorithm (AAA v.10.0.28) [16,17] and grid size $0.25 \mathrm{~cm}$ will be used for dose calculation. Four, equispaced fields (box technique) will be used for designing the 3DCRT plan. In this case, $15 \mathrm{MV}$ energy photon beams will be established. For each beam, the static multi-leaf collimator (MLC) will be used for matching the therapeutic field to the shape of the tumour. For dynamic techniques such as IMRT and VMAT, 6 MV energy photon beams will be used. The main conditions of these treatment plans will be, respectively: (1) IMRT - the seven fields technique realized through the sliding window method and (2) VMAT - two arcs realized by RapidArc technique (VMAT technique available on the Varian machines). The IMRT and VMAT plans will be prepared according to two scenarios with and without the optimization of dose distribution in the hip plates. As a result, five reference plans for each anatomical representation will be prepared. To simulate the image-guided radiotherapy, ten specific recombinations (for each anatomical representation separately) reflecting possible changes in the volume and position of the phantom components will be simulated by the Bayesian network. The treatment plans will be realised in accordance with the rules of image-guided radiation therapy, respectively, for each anatomical representation and each of the ten possible recombinations of the phantom components. The primary guideline for positioning based on CBCT images will be the position of the tumour. Before every fraction of RT, the phantom will be scanned on CT in the same technical condition as used for treatment planning. The delivered doses for $\mathrm{n}$-th fraction will be recalculated for the actual (n-th) set of CT scans of the phantom. To check accuracy between the calculated and delivered dose, the calculated doses (average dose from the outline of measurement area) will be compared with the doses measured in the phantom using ionization chambers. The doses will be measured in the thirteen points located respectively: in hip plates (six points, three in left and three in right hip plate), bladder (two points), rectum (two points) and in a tumour volume (three points).This verification will be performed both for the evaluation of fractional dose and cumulative doses that are the sum of the doses from ten delivered fractions. The deformable image registration and dose mapping procedures will be realized by in-house software [18]. As a result, errors resulting from (a) the differences between the calculated and measured doses and (b) procedures of image deformation and dose mapping will be determined for the 3DCRT, IMRT and VMAT techniques. The discrepancies between calculated/ mapped and measured doses will be used to estimate the uncertainties of the doses obtained during the treatment plan preparation, which are conditioned by changes in anatomy during the radiotherapy. Taking into account the estimated uncertainty, the comparative analysis of the dose distribution in the hip plates will be performed for 3DCRT, IMRT and VMAT. The main goal of the analysis will be to establish relative differences between the doses cumulated in the hip plates during radiation therapy of gynaecological cancer delivered by different techniques of dose delivery.

Especially, the analysis of (i) difference between calculated and measured doses for each technique of dose delivery (Wilcoxon test); (ii) difference of image deformation and dose mapping procedures in relation to measured doses (Wilcoxon test) and (iii) relative difference of the dose distribution in the hip plates between all dose delivery techniques (Friedman ANOVA) will be performed. All tests will be performed at $\alpha=$ 0.05 . See figure 2 for the steps of the planned study. 


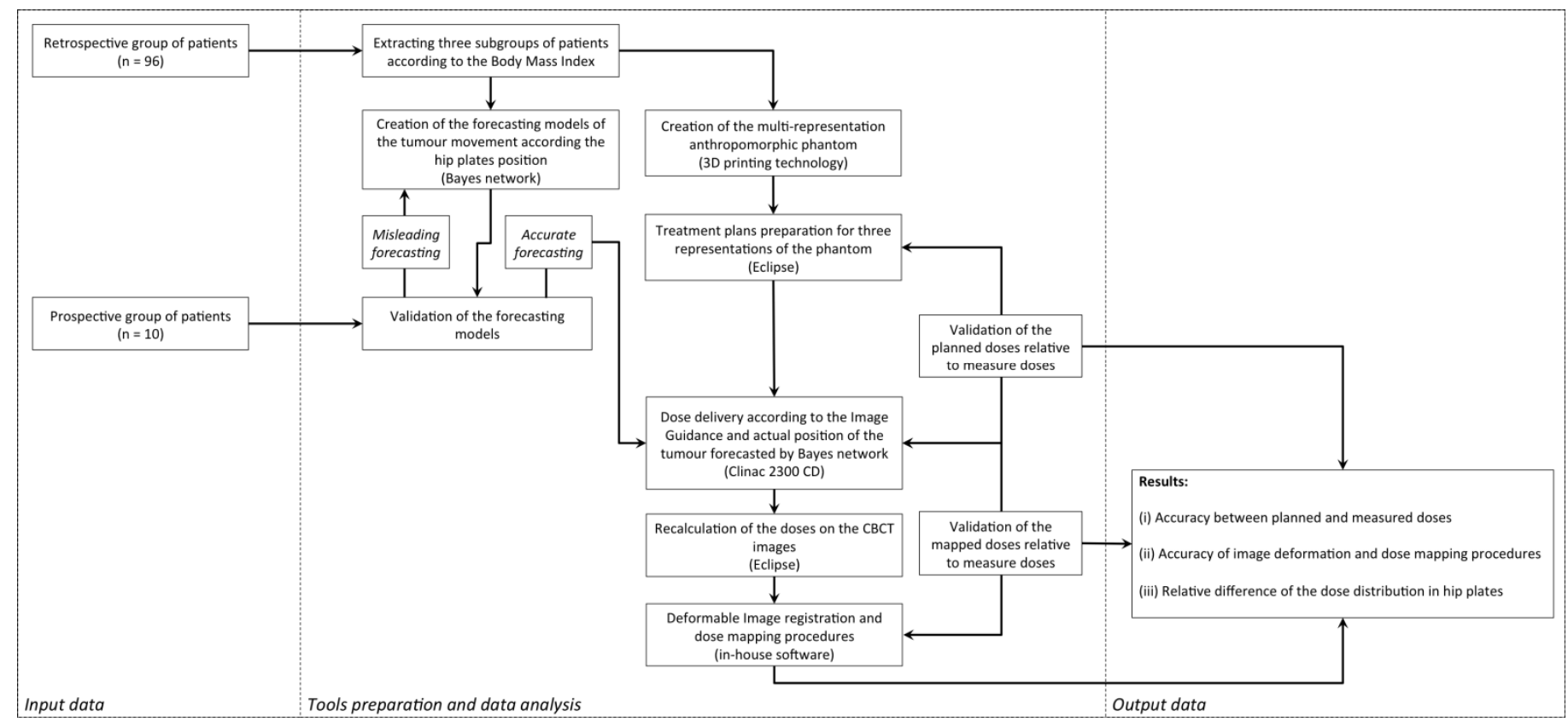

Figure 2. Scheme of the study.

\section{Discussion}

Lujan and collaborators [19] as well as Wong's team [5] showed that the application of IMRT allows dose reduction in the bone marrow area without interfering the homogeneity of doses in the tumour. Taking into consideration that $50 \%$ of the whole bone marrow is located in the hip plates [20], the reduction of the dose absorbed by the bone marrow may be expected to lower hematologic toxicity of the RT. Brixey et al. [8] reported significant reduction in acute hematologic toxicity for patients treated with IMRT in comparison to conventional RT. Nevertheless, the Brixey's results were obtained without regarding the bone marrow as an organ at risk at the stage of treatment planning and, thus, without undertaking any attempt to reduce dose in the bone marrow volume. Therefore, the outcome ought to be interpreted as an effect of a more conformal dose distribution for IMRT than for conventional RT.

Mell et al. [9] conducted a planned study in which they analysed the dose absorbed by the bone marrow during the course of radiotherapy and simultaneous chemotherapy (cisplatin; 40mg/ $/ \mathrm{m}^{2} /$ week). The outcome revealed that together with dose reduction in the bone marrow acute hematologic toxicity also falls and, consequently, the likelihood of a complete realization of chemotherapy increases. Nevertheless, this study shows clinical results in the light of the dose distribution in the hip plates that were gathered from initial treatment plans. Taking into account the possibility of changes in the position of the tumour, real dose delivered to the hip plates during image guided RT can be different than planned dose.

The hematologic toxicity reduction was observed for both optimisation schemes assuming a conscious dose reduction in the bone marrow and the optimisation schemes disregarding such a reduction. Therefore, the correlation between hematologic toxicity and optimisation scheme used for IMRT remains unclear.

A small number of papers assessed potential benefits of VMAT in the light of IMRT. Cozzi et al. [7] conducted a comparative analysis of the distribution of doses obtained for the IMRT and the VMAT. The analysis encompassed dose distributions obtained for eight patients with cervical cancer. They demonstrated that the VMAT technique, in comparison to the IMRT, allows a substantial dose reduction in OARs such as the intestines, rectum and bladder while, at the same time, ensuring homogeneous dose distribution in the PTV. These findings suggest that the VMAT technique helps obtain more conformal treatment plans than the IMRT, which in clinical practice may lead to even greater reductions of treatment toxicity. Nevertheless, the study did not analyse dose distribution obtained for the bone marrow and was limited only to a group of patients with cervical carcinoma. Therefore, it has not been verified so far whether the use of VMAT techniques ensures lower haematological toxicity compared to IMRT.

In the proposed study, relative differences between the doses cumulated in the hip plates during radiation therapy of gynaecological cancer realized by 3DCRT, IMRT and VMAT techniques of dose delivery will be established. The results will form the basis of the prospective clinical trial that will be designed for the assessment of hematologic toxicity and its correlation with the doses cumulated in the hip plates, for gynaecologic patients undergoing radiation therapy.

\section{List of Abbreviations}

RT - radiation therapy, 3DCRT - three-dimensional conformal radiation therapy, AP-PA - anterior/posterior - posterior/ anterior, OAR - organ at risk, PTV - planning target volume, IMRT - intensity modulated radiation therapy, VMAT volumetric modulated arc therapy, IGRT - image guided 
radiation therapy, BMI - body mass index, CT - computed tomography, CBCT - cone beam computed tomography, MLC - multi leaf collimator

\section{Acknowledgements}

Study was supported by institutional grant: wco 16/2014(75).

\section{References}

[1] Thwaites DI, Malicki J. Physics and technology in ESTRO and in Radiotherapy and Oncology: past, present and into the 4th dimension. Radiother Oncol. 2011;100(3):327-332.

[2] Fernandez-Ots A, Crook J. The role of intensity modulated radiotherapy in gynecological radiotherapy: Present and future. Rep Pract Oncol Radiother. 2013;18(6):363-370.

[3] Jodda A, Urbanski B, Piotrowski T, et al. Zależność pomiędzy zastosowaniem technik napromieniania w radioterapii a uzyskanym rozkładem dawek w raku szyjki lub trzonu macicy - przegląd literatury. Zeszyty Naukowe WCO (Letters in Oncology Science). 2013;10(4):88-92.

[4] Roeske JC, Lujan A, Rotmensch J, et al. Intensity-modulated whole pelvis radiation therapy in patients with gynecologic malignancies. Int J Radiat Oncol Biol Phys. 2000;48(5):1613-1621.

[5] Wong E, D'Souza DP, Chen JZ, et al. Intensity-modulated arc therapy for treatment of high-risk endometrial malignancies. Int $\mathbf{J}$ Radiat Oncol Biol Phys. 2005;61(3):830-841.

[6] Heron DE, Gerszten K, Selvaraj RN, et al. Conventional 3D conformal versus intensity-modulated radiotherapy for the adjuvant treatment of gynecologic malignancies: a comparative dosimetric study of dose-volume histograms. Gynecol Oncol. 2003;91(1):3945 .

[7] Cozzi L, Dinshaw KA, Shrivastava SK, et al. Treatment planning study comparing volumetric arc modulation with RapidArc and fixed field IMRT for cervix uteri radiotherapy. Radiother Oncol. 2008;89(2):180-191.

[8] Brixey CJ, Roeske JC, Lujan AE, et al. Impact of intensity modulated radiation therapy on acute hematologic toxicity in women with gynecologic malignancies. Int J Radiat Oncol Biol Phys. 2002; 54(5):1388-1396.

[9] Mell LK, Kochanski JD, Roeske JC, et al. Dosimetric predictors of acute hematologic toxicity in cervical cancer patients treated with concurrent cisplatin and intensity modulated pelvic radiation. Int J Radiat Oncol Biol Phys. 2005;63:S96-S97.

[10] Keys A, Fidanza F, Karvonen MJ, et al. Indices of relative weight and obesity. J Chronic Diseases. 1972;25(6):329-343.

[11] Su S, Moran K, Robar JL. Design and production of 3D printed bolus for electron radiation therapy. J Appl Clin Med Phys. 2014;15(4):194-211.

[12] Rengier F, Mehndiratta A, von Tengg-Kobligk H, et al. 3D printing based on imaging data: review of medical applications. Int $\mathbf{J}$ CARS. 2010;5(4):335-341.

[13] Jensen FV, Nielson TD. Bayesian Networks and Decision Graphs. New York: Springer-Verlag; 2007.

[14] Cowell RG, Dawid AP, Lauritzen AL, Spiegelhalter DJ. Probabilistic Networks and Expert Systems. New York: Springer; 1999.

[15] Murphy K. The bayes net toolbox for matlab. Computing Science and Statistics. 2001;3(2)3:1024-1034.

[16] Van Esch A, Tillikainen L, Pyykkonen J, et al. Testing of the analytical anisotropic algorithm for photon dose calculation. Med Phys. 2006;33(11):4130-4148.

[17] Fogliata A, Nicolini G, Vanetti E, et al. Dosimetric validation of the anisotropic analytical algorithm for photon dose calculation: fundamental characterization in water. Phys Med Biol. 2006;51(6):1421-1438.

[18] Piotrowski T, Ryczkowski A, Kazmierska J. B-spline registration based on new concept of an intelligent masking procedure and GPU computations for the head and neck adaptive tomotherapy. Technol Cancer Res Treat. 2012;11(3):257-266.

[19] Lujan AE, Mundt AJ, Yamada D, Rotmensch J, Roeske JC: Intensity-modulated radiotherapy as a means of reducing dose to bone marrow in gynecologic patients receiving whole pelvic radiotherapy. Int J Radiat Oncol Biol Phys. 2003;57(2):516-521.

[20] Ellis RE. The distribution of active bone marrow in the adult. Phys Med Biol. 1961;5(3):255-258. 